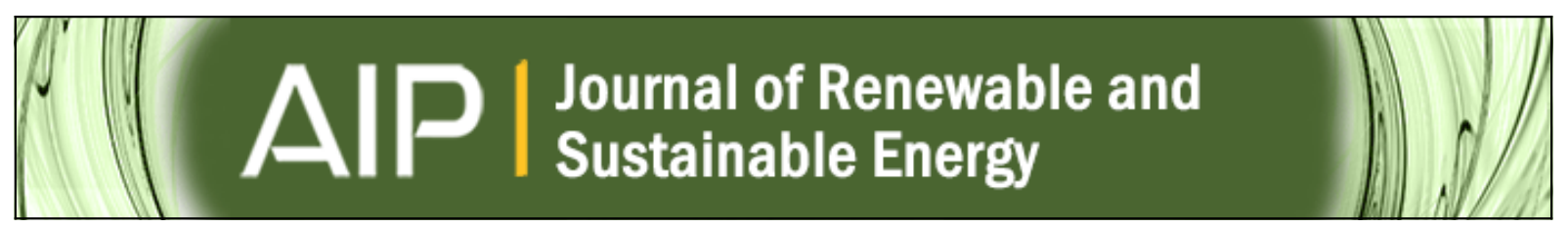

\title{
Theoretical analysis of GaAs/AIGaAs quantum dots in quantum wire array for intermediate band solar cell
}

Tomah Sogabe, Toshiyuki Kaizu, Yoshitaka Okada, and Stanko Tomi

Citation: Journal of Renewable and Sustainable Energy 6, 011206 (2014); doi: 10.1063/1.4828359

View online: http://dx.doi.org/10.1063/1.4828359

View Table of Contents: http://scitation.aip.org/content/aip/journal/jrse/6/1 ?ver=pdfcov

Published by the AIP Publishing

\section{Articles you may be interested in}

Effect of spacer layer thickness on multi-stacked InGaAs quantum dots grown on GaAs (311)B substrate for application to intermediate band solar cells

J. Appl. Phys. 111, 074305 (2012); 10.1063/1.3699215

Publisher's Note: "Structural atomic-scale analysis of GaAs/AIGaAs quantum wires and quantum dots grown by droplet epitaxy on a (311)A substrate" [Appl. Phys. Lett. 98, 193112 (2011)]

Appl. Phys. Lett. 99, 139901 (2011); 10.1063/1.3633014

Structural atomic-scale analysis of GaAs/AIGaAs quantum wires and quantum dots grown by droplet epitaxy on a (311)A substrate

Appl. Phys. Lett. 98, 193112 (2011); 10.1063/1.3589965

Intermediate-band material based on GaAs quantum rings for solar cells

Appl. Phys. Lett. 95, 071908 (2009); 10.1063/1.3211971

Absorption characteristics of a quantum dot array induced intermediate band: Implications for solar cell design Appl. Phys. Lett. 93, 263105 (2008); 10.1063/1.3058716

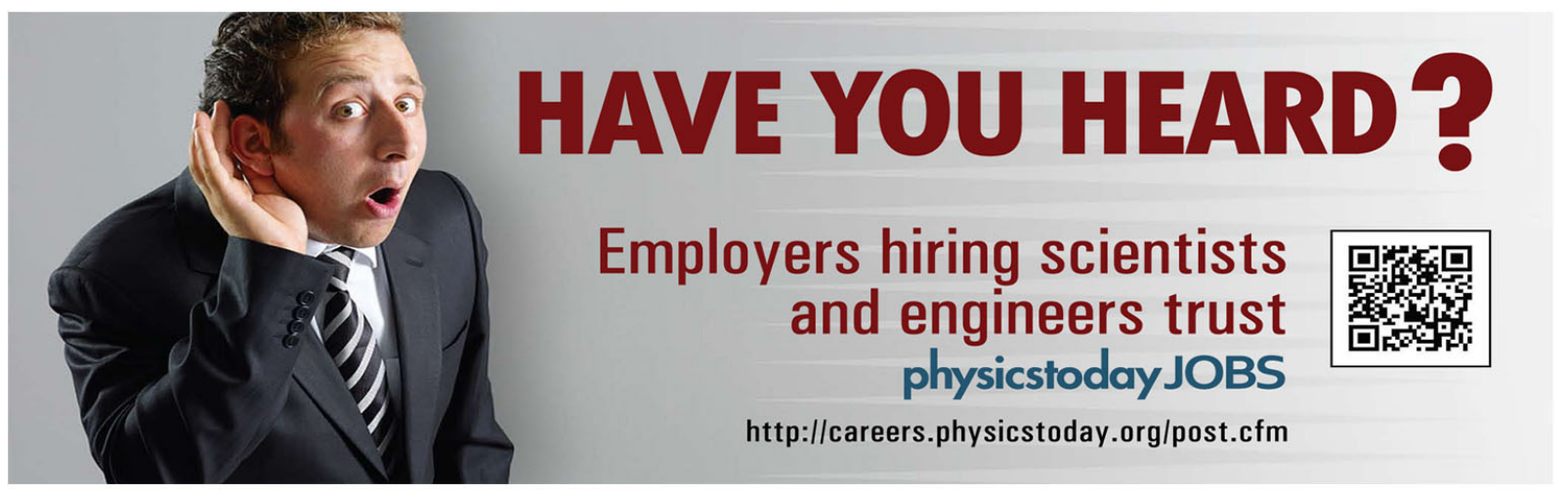




\title{
Theoretical analysis of GaAs/AIGaAs quantum dots in quantum wire array for intermediate band solar cell
}

\author{
Tomah Sogabe, ${ }^{1}$ Toshiyuki Kaizu, ${ }^{1}$ Yoshitaka Okada, ${ }^{1}$ and Stanko Tomić ${ }^{2, a)}$ \\ ${ }^{1}$ Research Center for Advanced Science and Technology, The University of Tokyo, \\ 4-6-1 Komaba, Meguro-ku, Tokyo 153-8904, Japan \\ ${ }^{2}$ Joule Physics Laboratory, School of Computing, Science, and Engineering, \\ University of Salford, Manchester M5 4WT, United Kingdom
}

(Received 31 May 2013; accepted 1 August 2013; published online 12 November 2013)

\begin{abstract}
A GaAs quantum dot (QD) array embedded in a AlGaAs host material was fabricated using a strain-free approach, through combination of neutral beam etching and atomic hydrogen-assisted molecular beam epitaxy regrowth. In this work, we performed theoretical simulations on a GaAs/AlGaAs quantum well, GaAs QD and QD array based intermediated band solar cell (IBSC) using a combined multiband $\mathbf{k} \cdot \mathbf{p}$ and drift-diffusion transportation method. The electronic structure, IB band dispersion, and optical transitions, including absorption and spontaneous emission among the valence band, intermediate band, and conduction band, were calculated. Based on these results, maximum conversion efficiency of GaAs/AlGaAs QD array based IBSC devices were calculated by a drift-diffusion model adapted to IBSC under the radiative recombination limit. (C) 2013 AIP Publishing LLC. [http://dx.doi.org/10.1063/1.4828359]
\end{abstract}

\section{INTRODUCTION}

Intermediate band solar cells (IBSC) concept have recently drawn a lot of attention, due to the reported high conversion efficiency of $63 \%$ under radiative recombination limit and maximum light concentration. ${ }^{1,2}$ In order to reach this theoretical efficiency limit, the optimal location of the intermediate band (IB) between the valence band (VB) and conduction band (CB) of the host materials is required to be $E_{g}(V B, C B)=1.9 \mathrm{eV}$ and $E_{g}(I B, C B)=0.7 \mathrm{eV}$. To date, a great number of material combinations have been employed to fabricate IBSCs. Among them, self-assembled InAs quantum dot (QD) arrays fabricated by molecular beam epitaxy (MBE) have been widely studied as a building block to create the IB in the GaAs host material. However, the band gap combination and the location of IB in InAs/GaAs QD based IBSCs is not favourable and the upper limit efficiency is around $20 \%$ ( 1 sun) and $34 \%$ (1000 suns). ${ }^{3-7}$ In order to improve the IBSC efficiency, host materials with much wider band gap such as $\mathrm{AlGaAs}$ or GaP are required. Recently, we have succeeded in the fabrication of GaAs QD arrays embedded in AlGaAs quantum-wire (QWR) host material by using a combination of neutral beam etching and atomic hydrogen-assisted MBE regrowth. ${ }^{8}$ This top-down lithography and etching method is a strain-free approach, with the advantages of being able to precisely control the size, spacing, and arrangement of the QD during growth, which is difficult to achieve by self-assembling growth. In this study, we performed theoretical simulation of GaAs/AlGaAs QD arrays using a combined multi band $\mathbf{k} \cdot \mathbf{p}$ and drift-diffusion transport method. The electronic structure, IB band dispersion, and optical transitions, including absorption and spontaneous emission among the VB, IB, and $\mathrm{CB}$, were calculated. Based on these parameters, the theoretical conversion efficiency limit of GaAs/AlGaAs QD array based IBSC devices were calculated by a drift-diffusion model adapted to IBSC. ${ }^{9-11}$

${ }^{\text {a)} E l e c t r o n i c ~ m a i l: ~ s . t o m i c @ s a l f o r d . a c . u k ~}$ 


\section{THEORETICAL MODEL}

The QD array model considered here consists of GaAs QDs. As shown in Fig. 1, in order to better simulate the realistic, experimentally grown, structures, we have used the experimental results for structural parameters. A GaAs quantum well embedded in an $\mathrm{Al}_{0.3} \mathrm{Ga}_{0.7} \mathrm{As}$ matrix was used as a control sample. For the QD array, we have chosen a QD of height $4 \mathrm{~nm}$, with vertical spacing set to $d_{z}=3 \mathrm{~nm}$. The quantum mechanical description of the IBSC was based on the solutions of the Schrödinger equation, $\hat{H}_{\mathbf{k} \cdot \mathbf{p}} \psi_{n}(\mathbf{r})=E_{n} \psi_{n}(\mathbf{r})$, with multi-band $\mathbf{k} \cdot \mathbf{p}$ Hamiltonian

$$
\hat{H}_{\mathbf{k} \cdot \mathbf{p}}=\frac{p^{2}}{2 m_{0}}+V(\mathbf{r})+\frac{\hbar^{2} k^{2}}{2 m_{0}}+\frac{\hbar \mathbf{k} \cdot \mathbf{p}}{m_{0}}+\frac{1}{4 m_{0}^{2} c^{2}}[\sigma \times \nabla V(\mathbf{r})](\mathbf{k}+\mathbf{p})+\mathbf{D}^{\varepsilon} \cdot \varepsilon+V_{\mathrm{PZ}}(\varepsilon),
$$

where the first term is the electron kinetic energy of the unperturbed system, the second is the potential due to QD shape and band edges of the QD or bulk material, and the rest are perturbative elements: the kinetic energy, coupling between zones in the system (the $\mathbf{k} \cdot \mathbf{p}$ term), the spin orbit split off energy, the Pikus-Bir strain Hamiltonian, and the piezoelectric potential, respectively. The whole Hamiltonian, $\hat{H}_{\mathbf{k} \cdot \mathbf{p}}$, is expanded using only the $s$-anti-bonding and $p_{x}, p_{y}, p_{z^{-}}$ bonding orbitals, all spin degenerate, into so called the 8-band $\mathbf{k} \cdot \mathbf{p}$ Hamiltonian. The wave functions, $\psi_{n}(\mathbf{r})$, are expanded into the plane-wave basis, $|\mathbf{k}+\mathbf{K}\rangle=e^{i(\mathbf{k}+\mathbf{K}) \mathbf{r}}$, where $\mathbf{K}$ is the wave vector of the QD array, in order to provide the periodic boundary conditions suitable for the analysis of such structures. More details about methodology can be found elsewhere. ${ }^{11,13}$

From the $\mathbf{k} \cdot \mathbf{p}$ calculation, we obtained the position of $E_{L}, E_{H}$, and information about the density of states of $N_{V B}, N_{C B}$, and $N_{I B}$. With the energy levels and wave functions, following the dipole approximation, the absorption coefficient is given as

$$
\alpha(\hbar \omega)=\frac{\pi e^{2}}{c \epsilon_{0} m_{0}^{2} \bar{n} \omega \Omega} \sum_{\mathbf{K}} \sum_{i, f}|\langle i|\hat{\mathbf{e}} \cdot \mathbf{p}(\mathbf{K})| f\rangle|^{2} \delta\left[E_{f}(\mathbf{K})-E_{i}(\mathbf{K})-\hbar \omega\right]\left(f_{i}-f_{f}\right),
$$

where $\hbar$ is the reduced Planck constant, $\omega$ is the photon frequency, $e$ is the electron charge, $\bar{n}$ is the refractive index, $\epsilon_{0}$ is the dielectric permittivity of a vacuum, $c$ is the vacuum speed of light, $\Omega$ is the volume of the structure, $\hat{\mathbf{e}}$ is the unit vector of light polarization, and $\mathbf{p}(\mathbf{K})$ is the momentum operator. The optical dipole matrix element is given by $\langle i|\hat{\mathbf{e}} \cdot \mathbf{p}(\mathbf{K})| f\rangle$, and varies inside the Brillouin zone (BZ) of an QD array. ${ }^{9}$ The initial and final state energies are $E_{i}(\mathbf{K})$ and $E_{f}(\mathbf{K})$, which also vary throughout the QD array BZ, $\hbar \omega$ is the photon energy, and $f_{i(f)}$ is the initial (final) state Fermi-Dirac distribution function.

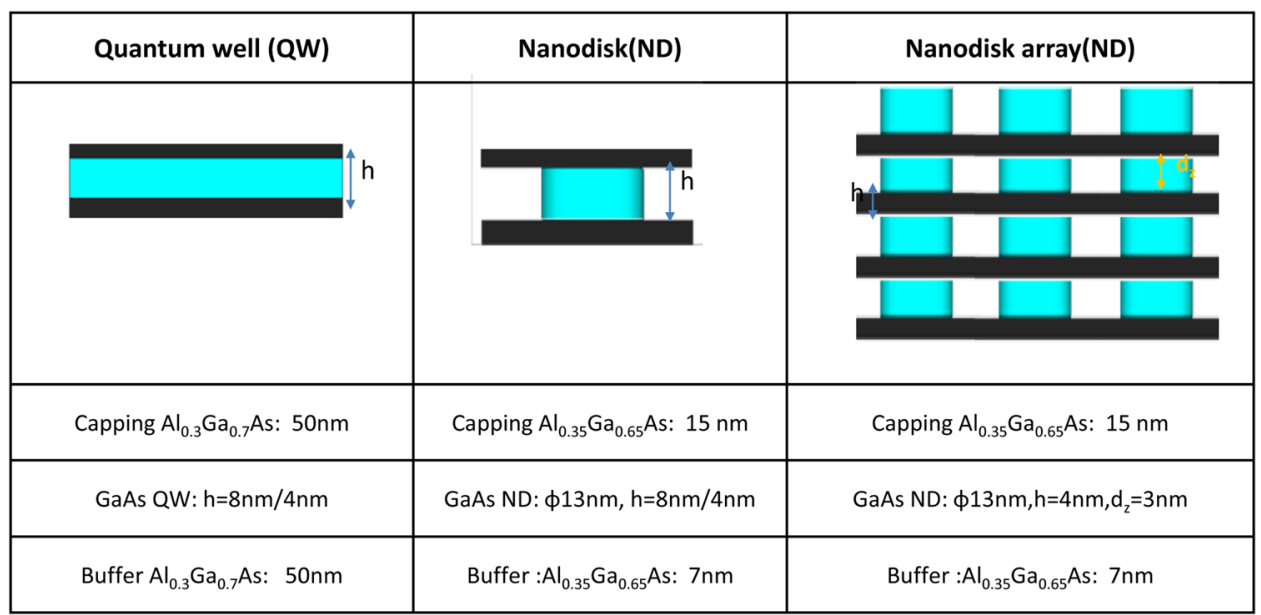

FIG. 1. Structure parameters used in the multiband k.p and drift-diffusion simulation for GaAs quantum well, GaAs Nanodsik, and GaAs Nanodisk array. 
The characteristic function for a QD of arbitrary shape, $\Sigma$, can be expressed in reciprocal space as ${ }^{12-14}$

$$
\chi(\mathbf{k})=\frac{1}{(2 \pi)^{3}} \int_{\Sigma} \exp (-i \mathbf{k} \cdot \mathbf{r}) d \mathbf{r}
$$

The disk like shape of the QD in a quantum wire structure, used in our analysis, in the reciprocal space suitable for modelling periodic arrays becomes

$$
\chi_{\text {disk }}(\mathbf{k})=\frac{2 \pi i}{\mathbf{k}_{\|} k_{z}}\left[e^{-i k_{z} H}-1\right] D J_{1}\left(\mathbf{k}_{\|} D\right),
$$

where $\mathbf{k}_{\|}=k_{x} \hat{x}+k_{y} \hat{y}$ is the in-plane wave-vector, $k_{z}$ the wave-vector in the $z$-direction, $J_{1}$ is the Bessel function of the first kind, and $D$ and $H$ are the diameter and height of the quantum nano-disk, respectively.

\section{RESULTS}

\section{A. Band edge profile of GaAs/AIGaAs QD}

Figure 2 shows the two dimensional band edge profile of the GaAs/AlGaAs QD, with $D=15 \mathrm{~nm}$ and $H=4 \mathrm{~nm}$. In the simulation we have used a shape function, Eq. (4), in reciprocal space to describe the QD geometric structure. Figure 2 shows the CB and heavy hole $(\mathrm{HH})$ band edge profile of the GaAs QD projected in the $x y$-plane $(\mathrm{c}, \mathrm{d})$ and $y z$-plane $(\mathrm{a}, \mathrm{b})$. The number of Fourier components was set as $N_{x}=N_{y}=N_{z}=50$. The CB band edge is found at around $1.688 \mathrm{eV}$ with the band offset $\mathrm{CB}=0.19 \mathrm{eV}$, as shown in Figures 2(a) and 2(c). The $\mathrm{HH}$ edge is located at $0.018 \mathrm{eV}$ with $\mathrm{HH}=0.137 \mathrm{eV}$. These values are fairly close to the preset $\mathrm{CB}$ edge value $1.678 \mathrm{eV}$ with $\mathrm{CB}=0.16 \mathrm{eV}$ and $\mathrm{HH}$ edge value $0.0 \mathrm{eV}$ with $\mathrm{HH}=0.124 \mathrm{eV}$.
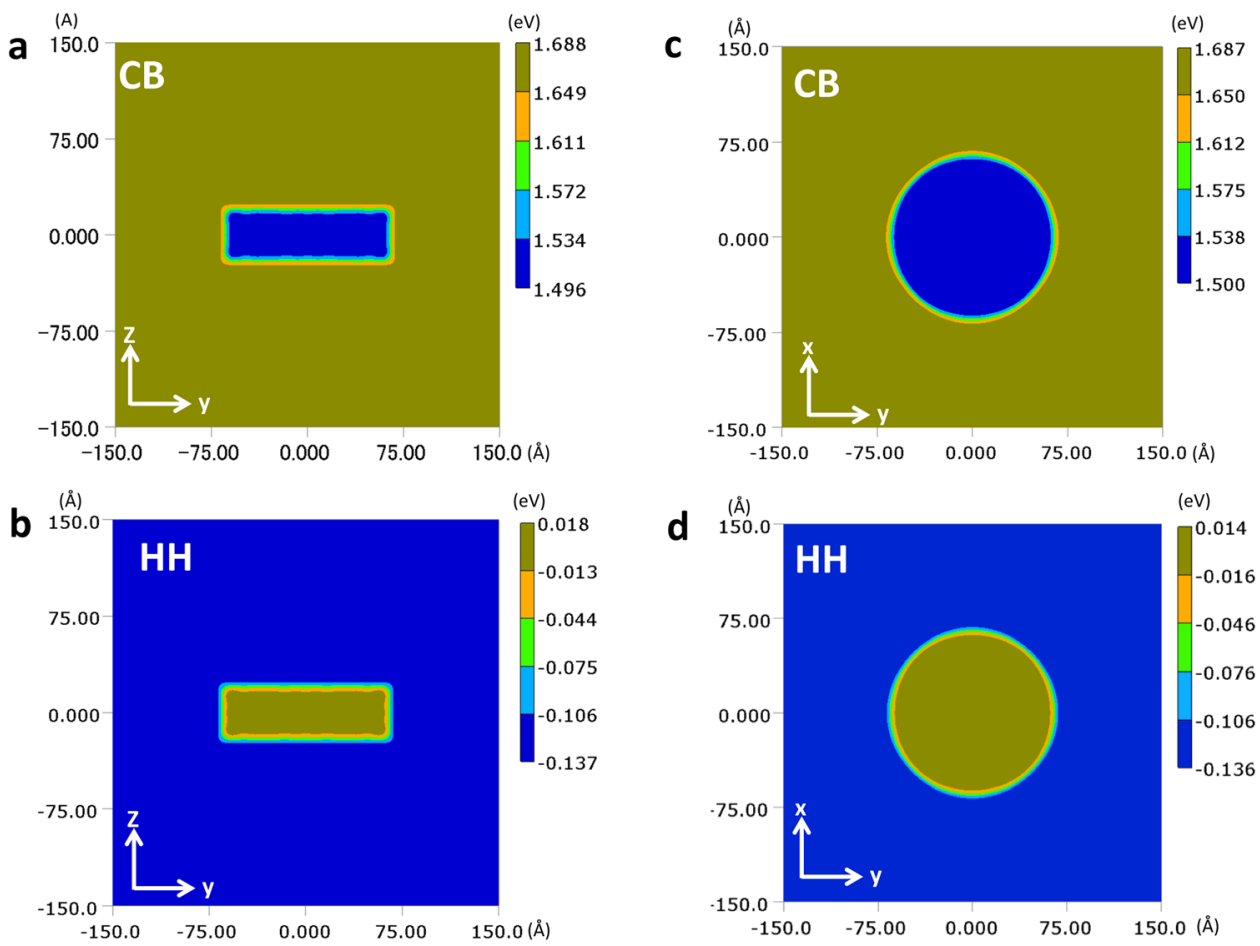

FIG. 2. Band edge profile for GaAs QD: (a) CB edge profile in $y z$ plane, (b) HH band edge profile in $y z$ plane, (c) CB edge profile in $x y$ plane, (d) $\mathrm{HH}$ band edge profile in $x y$ plane. The Fourier components used in the simulation are $N_{x}=N_{y}=N_{z}=50$. 


\section{B. Electronic and optical properties of GaAs QW and GaAs QD}

Figure 3(a) shows the subband dispersions, along in-plane wave-vector $\mathbf{k}_{\|}$, of the GaAs QW embedded in $\mathrm{Al}_{0.3} \mathrm{Ga}_{0.7} \mathrm{As}$ barrier material. The well width is $4 \mathrm{~nm}$. It can be clearly seen here that nonparabolic (warping) subband dispersion curves exist in the valence band, which reflects the band mixing behavior in quantum wells due to the strong coupling between heavy-hole $(\mathrm{HH})$, light-hole (LH) and spin-orbit split-off (SO) subbands in the VB. The valence band mixing has a profound impact on the calculation of the absorption or gain spectrum in a quantum well. Figure 3(b) shows the absorption spectrum calculated between the optically allowed transitions of $\mathrm{CB}$ and VB. In order to fit the experimental results, we have also calculated the absorption spectra under a two-dimensional exciton model.

The spontaneous emission spectrum was calculated using the following formula: ${ }^{15-18}$

$$
R_{s p}(E)=\left(\frac{8 \pi \bar{n} E^{2}}{h^{3} c^{2}}\right) \frac{1}{e^{\frac{E-\Delta F}{k_{B} T}}-1} \alpha(E)
$$

where $h$ is the Planck constant, $c$ is the speed of the light in a vacuum, $\bar{n}$ is the refractive index of GaAs, $\alpha(E)$ is the absorption coefficient, Eq. (2), $k_{B}$ is the Boltzmann constant, $T$ is the temperature, and $\Delta F$ is the separation of the quasi-Fermi levels. The key step to calculate spontaneous emission is to accurately determine the $\Delta F$. Near the $\mathrm{BZ}$ center, a parabolic band approximation is valid for QW. Meanwhile, if assuming that electrons occupy the ground state of both $\mathrm{CB}$ and $\mathrm{VB}$, the injected carrier density can be expressed as, $N_{e}=\left(m^{*} k_{B} T\right) /\left(\pi \hbar^{2} L_{Q W}\right) \ln \left[1+e^{\left(F_{c}-E_{C B 1}\right) / k_{B} T}\right]$. Here, $L_{Q W}$ is the QW width, $m^{*}$ is the electron effective mass, and the quasi-Fermi level for electron, $F_{c}$, can be calculated as $F_{c}=E_{C B 1}+k_{B} T\left(e^{N_{e} L_{Q W} / N_{s}}-1\right)$, where $N_{s}=\left(m^{*} k_{B} T\right) /\left(\pi \hbar^{2}\right)$. The quasi-Fermi level for hole, $F_{v}$, can be determined similarly as $F_{v}=E_{H H 1}-k_{B} T\left(e^{N_{h} L_{Q W} / N_{s}}-1\right)$. Due to negligible difference between perpendicular and in-plane electron effective mass in GaAs, we adopted the effective bulk mass $m^{*}=0.067 m_{0} \cdot{ }^{19,20}$ For a doped material, the charge neutrality relation
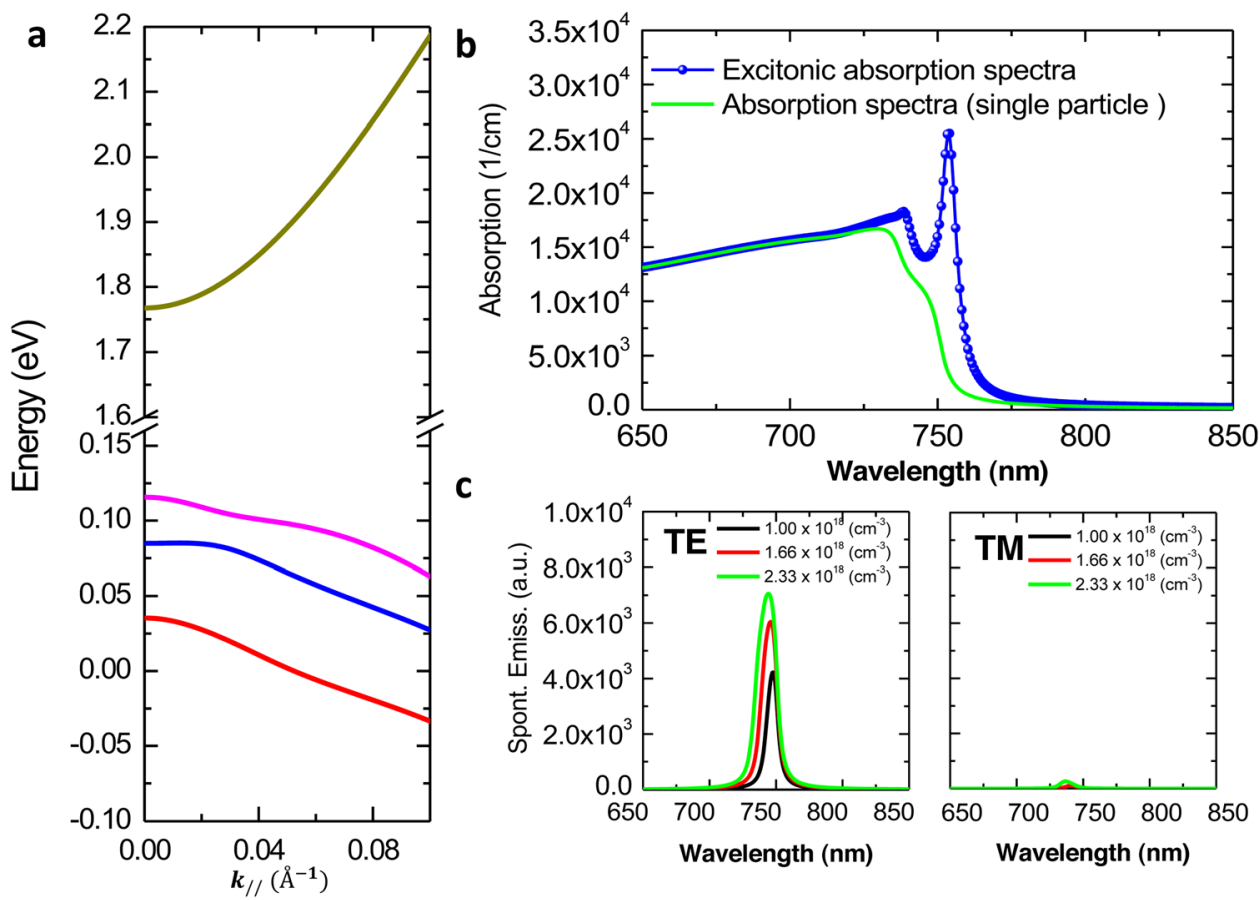

FIG. 3. Optical absorption and spontaneous emission spectrum for GaAs QW. (a) Band dispersion of GaAs QW (4 nm thickness) along $K_{z}$ direction. (b) Optical absorption spectra calculated under exciton model and single particle model. (c) Spontaneous emission spectra calculated under TE and TM different polarization modes. Three injected carrier concentrations were employed to fit the experimental results. 
$N_{h}=N_{e}+N_{A}-N_{D}$ holds and for an undraped QW, $N_{h}$ is simply equal to $N_{e}$. In our simulation, we have examined three different carrier sheet densities: $N_{e}=1 \times 10^{18}, 1.66 \times 10^{18}$, and $2.33 \times 10^{18} \mathrm{~cm}^{-2}$. Taking $N_{s}=2 \times 10^{11} \mathrm{~cm}^{-2}$ for the $4 \mathrm{~nm}$ wide GaAs QW structure, we can obtain the following values for $\Delta F: 1.65+0.034 \mathrm{eV}, 1.65+0.102 \mathrm{eV}$, and $1.65+0.311 \mathrm{eV}$ for the three sheet densities, respectively. Assuming a finite broadening in the density of states of the QW, the final spontaneous emission spectrum is obtained, as shown in Figure 3(c). It is found here again that the TM polarization gives rise to weak emission spectra. This can be quantitatively explained by the shape anisotropic effect of the wave function in the QW. Fitting of experimental PL data is plotted in Figure 4(b). The emission spectra, including both TE and $\mathrm{TM}$ polarization, were averaged by $(2 \mathrm{TE}+\mathrm{TM}) / 3$. The simulation and experimental results are consistent to each other. The slighter larger value of FWHM (full width at half maximum) is due to the larger broadening factor used in the simulation and can be effectively tailored to match the experimental results. We also plotted experimental and simulated results for the QW with $8 \mathrm{~nm}$ width for better comparison.

In order to compare with the experimental PL results, we carried out a detailed calculation of the spontaneous emission spectra of the GaAs QD. Similar to the QW case, separation of quasi-Fermi levels $\Delta F=F_{c}-F_{v}$ has to be estimated first. The quasi-Fermi levels for electron and hole in GaAs QD is determined by the following formulae: ${ }^{16}$

$$
\begin{aligned}
& n_{2 D}=\frac{2 N_{Q D}^{2 D}}{H} \int_{0}^{\infty} \frac{1}{\sqrt{2 \pi} \sigma_{c}} e^{-\left(E-E_{C B 1}\right)^{2} / 2 \sigma_{c}^{2}} \frac{1}{1+e^{\left(E-F_{c}\right) / k_{B} T}} d E, \\
& p_{2 D}=\frac{2 N_{Q D}^{2 D}}{H} \int_{0}^{\infty} \frac{1}{\sqrt{2 \pi} \sigma_{v}} e^{-\left(E-E_{H H 1}\right)^{2} / 2 \sigma_{v}^{2}} \frac{1}{1+e^{\left(E-F_{v}\right) / k_{B} T}} d E,
\end{aligned}
$$

where $N_{Q D}^{2 D}$ is areal density of GaAs QDs and $n_{2 D}$ and $p_{2 D}$ are the electron and hole concentration normalised by the QD height, $H$. In order to better simulate the experimental results, we have taken the inhomogenous broadening effect due to the size fluctuations of the QDs into consideration. Carrier distribution is described by a Gaussian function centered at the discrete energy levels $E_{C B 1}$ and $E_{H H 1}$ with FWHM denoted as $\sigma_{c}$ and $\sigma_{v}$, where $\sigma_{c}$ is much larger than $\sigma_{v}$ due to large effective mass in VB (here $\sigma_{c}=0.025 \mathrm{eV}$ and $\sigma_{v}=0.0012 \mathrm{eV}$ ). If $n_{2 D}$ and $p_{2 D}$ are known, quasi-Fermi levels $F_{c}$ and $F_{v}$ can be determined from formulae Eqs. (6) and (7). Standard numerical simulation methods such as Newton-Raphson iteration are not suitable here. However, a simple "graphical method," by plotting $n_{2 D}$ or $p_{2 D}$ as a function of $F_{c}$ or $F_{v}$, can be used to determine the value of $F_{c}$ and $F_{v}$. Fig. 4(b) shows the simulated spontaneous emission
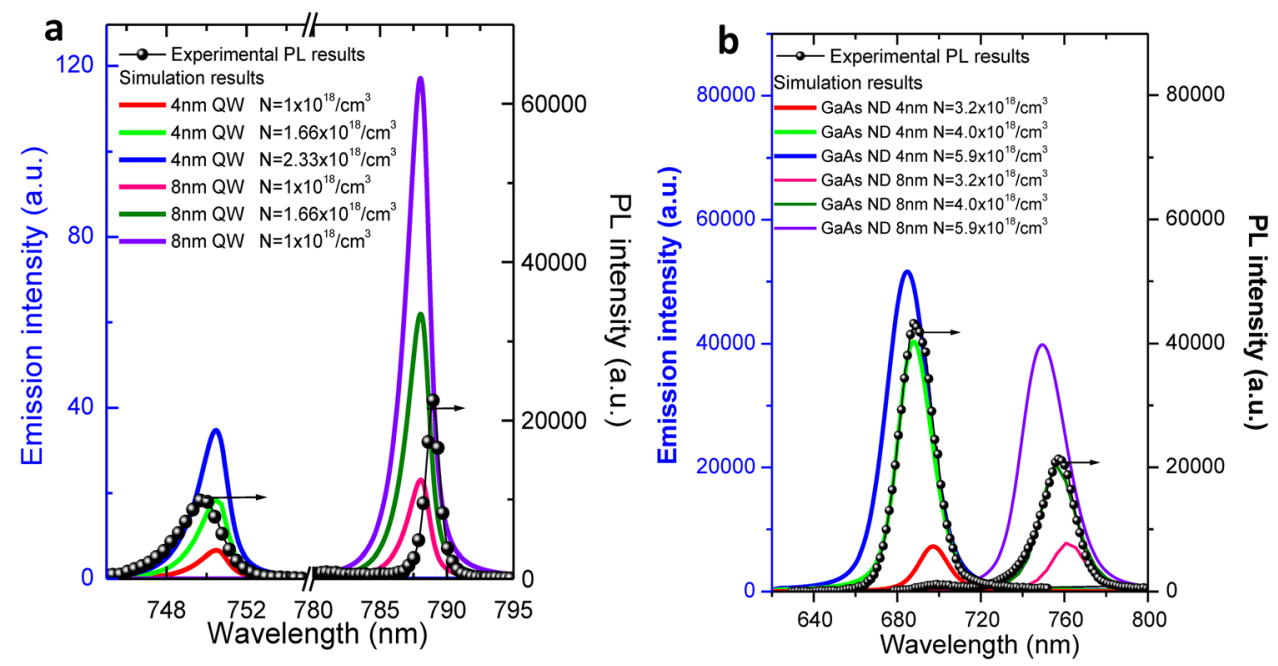

FIG. 4. Comparison of the simulated spontaneous emission spectrum with experimental PL results: (a) $4 \mathrm{~nm} / 8 \mathrm{~nm}$ GaAs QWs, (b) GaAs QDs with height of $4 \mathrm{~nm}$ and $8 \mathrm{~nm}$. Injection carrier concentration was varied to better fit the PL results. 

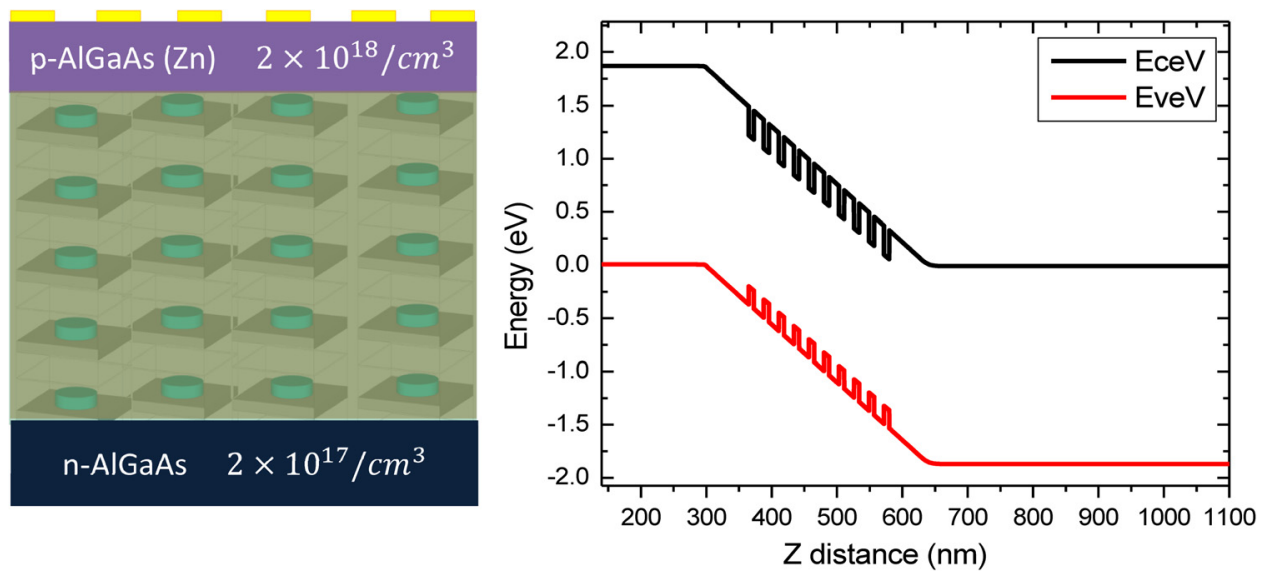

FIG. 5. Device structure model (left) and Poison equation derived potential profile (right) for AlGaAs/GaAs/AlGaAs $p-i-n$ solar cell device.

results along with the experimental PL results. From experimental fabrication conditions, we obtain the areal density of GaAs QD as $5 \times 10^{10} \mathrm{~cm}^{-2}$. We have chosen three different electron concentrations: $n_{3 D}=3.2 \times 10^{18} \mathrm{~cm}^{-3}, 4.0 \times 10^{18} \mathrm{~cm}^{-3}$, and $5.9 \times 10^{18} \mathrm{~cm}^{-3}$. The temperature was set at $30 \mathrm{~K}$. It is found that at an electron concentration of $4.0 \times 10^{18} \mathrm{~cm}^{-3}$, corresponding quasi-Fermi level separation is in good agreement with the experimental PL results.

\section{GaAs QD array based IBSC device simulation}

Figure 5 shows the device structure for the GaAs QD based IBSC. We have adopted a $p-i-n$ structure where in the $\mathrm{i}$ region, ten layers of GaAs QD were embedded in the $\mathrm{Al}_{0.3} \mathrm{Ga}_{0.7} \mathrm{As}$ barrie material. Band edge profile is presented in Figure 5(b). In order to obtain miniband information due to the vertical coupling of the ten-layer GaAs QD, we have performed band dispersion calculations assuming periodic boundary conditions. The initial structure simulation parameters were presented in Figure 1 (see the QD array). Compared to a QD of width $8 \mathrm{~nm}$, the wave function in QD of with $4 \mathrm{~nm}$ shows a stronger tendency to delocalize.

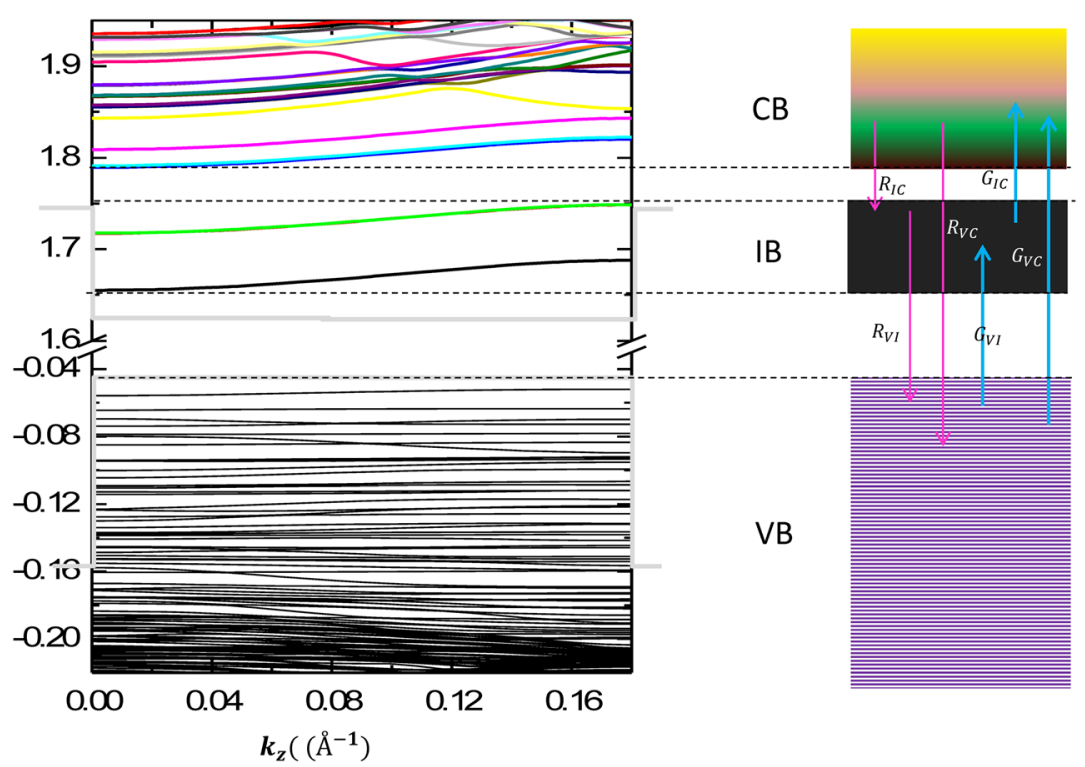

FIG. 6. Band dispersion for GaAs QD array. The height of GaAs QD is $4 \mathrm{~nm}$ and the inter-distance between neighbouring QD is set as $3 \mathrm{~nm}$. Only vertical coupling $\left(K_{z}\right)$ was taken into account. A sketched band model is shown on the right side. Three optical absorption and recombination paths are also presented. 
We therefore chose GaAs QD, of width $4 \mathrm{~nm}$ in the array simulation. Band dispersion along wave vector $K_{z}$ is presented in Figure 6. It can be seen that the band dispersion in VB is featureless due to the large effective mass of holes $(\mathrm{HH}, \mathrm{LH})$ which flatten the band dispersion. In contrary, we found two minibands in $\mathrm{CB}$, labeled, $e 1$ and $e 2$. Each has band width around $45 \mathrm{meV}$. However, since the band gap between these two bands is less than $25 \mathrm{meV}$, these two minibands are treated as one band by taking thermal fluctuation at room temperature $(300 \mathrm{~K})$ into consideration. A sketch of the band model for VB and IB is CB is plotted in the right side of Figure 6. After having obtained band width and positions, we calculated the optical absorption coefficients $\alpha_{v, i}, \alpha_{i, c}$, and $\alpha_{v, c}$ for the three transitions: $\mathrm{VB} \rightarrow \mathrm{IB}, \mathrm{IB} \rightarrow \mathrm{CB}$, and $\mathrm{VB} \rightarrow \mathrm{CB}$, respectively, which are shown in Figure 7. We also calculated the absorption spectrum from VB $\rightarrow(\mathrm{CB}+\mathrm{IB})$, which is shown in Fig. 7(d). This transition accounts for the case where the quasi-Fermi level in IB is not separable from $\mathrm{CB}$. In this case, we need to treat the IB and $\mathrm{CB}$ as one band. It should be noted that the broadening parameter has a strong effect on the magnitude of absorption coefficients. As we have mentioned before, the QD array studied here was fabricated using a top-down lithography and etching method, the advantage of which is the high accuracy in QD size control. Therefore, we used a fairly small broadening width of $10 \mathrm{meV}$ in the absorption coefficient calculation.

All information obtained from the electronic structure calculations were used with the driftdiffusion model, adapted for a IBSC. The main contents of the IBSC drift-diffusion model are given as follows. ${ }^{21-23}$ The generation rate of electrons per unit surface area from a band $i$ to a band $f$ higher in energy is denoted by $G_{i f}$. The recombination rate of electrons per unit surface area from a band $f$ to a band $i$ lower in energy is denoted by $R_{i f}$. For the intermediate band to be in equilibrium, the net result of all the transitions into and out of the band must be zero, i.e., $G_{v, i}-R_{v, i}+R_{i, c}-G_{i, c}=0$. Once a system that is in steady state is found, the corresponding current out of the conduction band can be evaluated: $J=e\left(G_{v, c}+G_{i, c}-R_{i, c}-R_{v, c}\right)$. The flux of photons per unit solid angle from a blackbody would be given by Planck's law if in a vacuum. We however will carry out all our analysis inside the cell, a material of refractive index $\hat{n}$, which due to Snell's law requires a different angular dependence. Inside a material of refractive index,

a
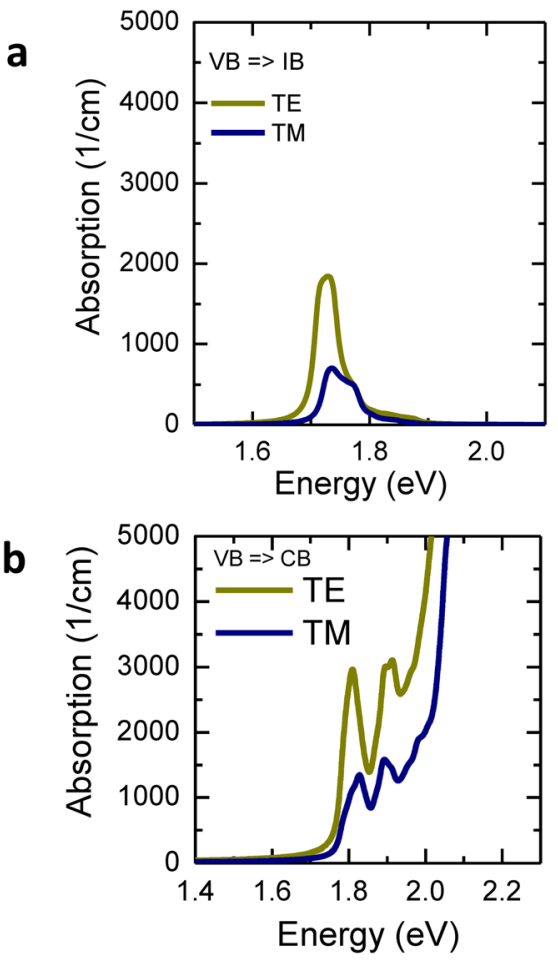

c

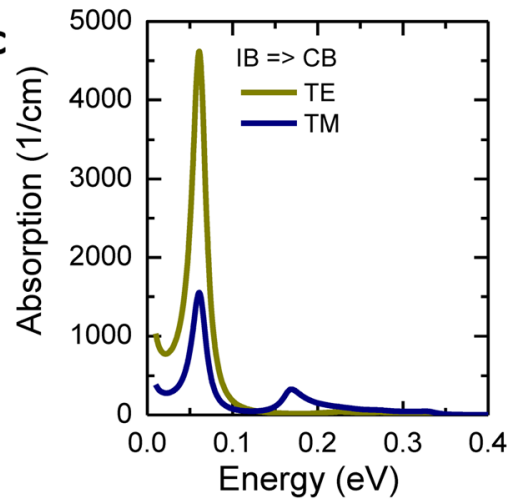

d

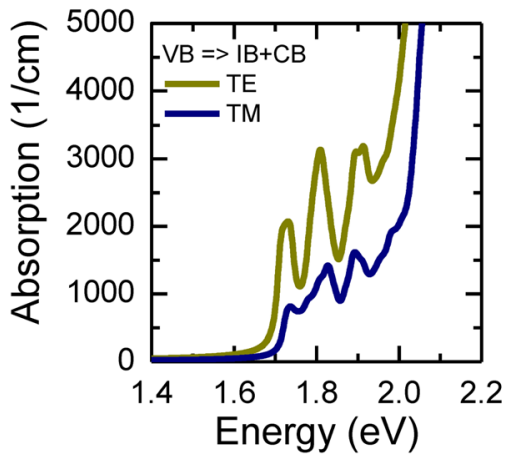

FIG. 7. Optical absorption coefficients for (a) VB to IB, (b) VB to CB, and (c) CB to IB transitions. We also calculated the continuous absorption from VB to (IB $+\mathrm{CB})$, as shown in $(\mathrm{d})$. 
the flux of photons per unit surface area, per unit solid angle, in the range $E \rightarrow E+d E$ from a blackbody at temperature $T$ is given as $\phi_{i f} d E=\left(2 \bar{n}^{2} / h^{3} c^{2}\right)\left(E^{2} / e^{E / k_{B} T}-1\right) d E$. In this model, we assumed non-overlapping, constant, absorption coefficients $\alpha_{i f}$. This means that the flux of photons able to cause a transition between any two energy levels $i$ and $f$ is given by

$$
\Phi_{i f}=\frac{2 \bar{n}^{2}}{h^{3} c^{2}} \int_{E_{\text {low }}}^{E_{\text {high }}} \frac{E^{2} d E}{e^{\frac{E}{k_{B} T}}-1}
$$

where $E_{\text {low }}$ is the energy gap corresponding to the transition $i \rightarrow f$ and $E_{\text {high }}$ is the next lowest energy gap. The flux from the sun is received outside the material up to an angle $\theta_{s}$ at a temperature of $6000 \mathrm{~K}$, and for angles larger than this the flux is received from the surroundings at a temperature of $300 \mathrm{~K}$. These angles must be converted using Schnell's law to angles inside the material $\theta_{s}$ and $\theta_{c}$ (critical angle of total internal reflection). Now, if we consider a point a distance $z$ from the surface of the solar cell, the generation follows Beer-Lambert's law but with two terms, one taking into account photons reflected off the back of the solar cell. However, the optical path traveled to our point clearly has an angular dependence, and so the generation rate per unit volume, $g_{\text {if }}$ involves an integral over $\theta$,

$$
g_{i f}=2 \pi \int_{0}^{\theta_{c}} \Phi_{i f}(\theta) \alpha_{i f}\left[\exp \left(-\alpha_{i f} \frac{z}{\cos \theta}\right)+\exp \left(-\alpha_{i f} \frac{2 W-z}{\cos \theta}\right)\right] \cos \theta \sin \theta d \theta .
$$

The angular dependence of $\Phi(\theta)$ takes care of the fact that some radiation comes from sun, $0<\theta<\theta_{s}$ and some from the ambient surroundings $\theta_{s}<\theta<\theta_{c}$. The rate per unit surface area is found by integrating over $z$, so that for a solar cell of width $W, G_{i f}=\int_{0}^{W} g_{i f} d z$.

This model differs from the traditional approach to the intermediate band solar cell by allowing both the absorption coefficients and the filling of the inter-mediate band to vary. They are coupled by assuming the absorption coefficients depend linearly on the carrier density in the intermediate band, i.e., $\alpha_{i c}=\sigma_{i c} n_{i b}, \alpha_{v i}=\sigma_{v i}\left(N_{i b}-n_{i b}\right)$, where $\sigma_{i c}$ is the absorption cross section for the transition, $n_{i b}$ is the electron concentration in the IB, and $N_{i b}$ is the density states in the IB. This is to say that the absorption coefficient into the IB is proportional to the concentration of empty states there, and the absorption coefficient out of the IB is proportional to the concentration of electrons in there.

The net rate $r_{i f}$ of electrons per unit volume, per unit solid angle, de-excited from a band $f$ to a band of lower energy $i$ whilst radiating into the same energy range of photons, which drives the corresponding absorption, is given by the generalised Planck formula,

$$
r_{i f}=\alpha_{i f} \frac{2 \bar{n}^{2}}{h^{3} c^{2}} \int_{E_{\text {low }}}^{E_{\text {high }}} \frac{E^{2} d E}{e^{\frac{\left(E-\mu_{i j}\right)}{k_{B} T}}-1},
$$

where $\mu_{i f}=F_{f}-F_{i}$ is the difference in energy of the Fermi levels, and $T$ is the temperature of the solar cell, i.e., $300 \mathrm{~K}$. Due to total internal reflection, this radiation can only escape the cell if it is emitted within a cone of semi-angle $\theta_{c}$, the critical angle of total internal reflection, either towards the front surface of the cell or the back surface of the cell (from which it will reflect). Since this emitted radiation may stimulated transitions along this optical path and be reabsorbed, we find an expression for the effective recombination rate per unit volume,

$$
\rho_{i f}=2 \pi \int_{0}^{\theta_{c}} r_{i f}\left(\mu_{i f}\right) \alpha_{i f}\left[\exp \left(-\alpha_{i f} \frac{z}{\cos \theta}\right)+\exp \left(-\alpha_{i f} \frac{2 W-z}{\cos \theta}\right)\right] \cos \theta \sin \theta d \theta .
$$

We note that $r_{i f}$ is angular independent and the rate per unit surface area is again found by the integral, $R_{i f}=\int_{0}^{W} \rho_{i f} d z$.

In the steady state relevant for SC operation, $\partial / \partial t=0$. We adopt the charge conservation rule $n+n_{i b}-p+N_{d}^{+}=0$, where $N_{d}^{+}$is the pre-fill donor concentration of IB. In the model, we 
have assumed that all donor atoms are ionized. The concentrations $n, p$, and $n_{i b}$ are given by the relevant Fermi-Dirac distributions: $n=N_{c} /\left[e^{\left(E_{c}-F_{c}\right) / k_{B} T}-1\right], p=N_{v} /\left[e^{\left(F_{v}-E_{v}\right) / k_{B} T}-1\right]$, and $n_{i b}=N_{i b} /\left[e^{\left(E_{i}-F_{i}\right) / k_{B} T}-1\right]$, where $N_{c}, N_{v}$, and $N_{i b}$ are the effective density of states in the respective band.

For a current extracted from the solar cell at voltage $V$, the split between the valence and conduction band Fermi-levels in electron-volts is equal to that voltage, i.e., $F_{c}-F_{v}=e V$. Figure 8 shows the efficiencies contour plot of a pre-filled IBSC with filling factor of 0.5 under light concentration of $\mathrm{X}=1000$ suns. At the subband gap combination of $E_{g}(V B, I B)=1.3 \mathrm{eV}$, and $E_{g}(I B, C B)=0.74 \mathrm{eV}$, a maximum efficiency of $57 \%$ is obtained. This value is slightly lower than the detailed balance limit because of the limited device thickness (here $5 \mu \mathrm{m}$ ). Setting the thickness large enough and under maximum concentration, we obtained exactly the $63.2 \%$, the idealised efficiency value reported before.

Next, we performed efficiency calculations for the GaAs QD using the density of states, absorption coefficients, band width and location obtained by multiband $\mathbf{k} \cdot \mathbf{p}$ calculation. The results are shown in Figure 9. Under the radiative recombination limit, we obtained $22.3 \%$ for a GaAs QD array based IBSC under 1 sun. Under 1000 suns, the efficiency can reach 33\%. In Figure 9, the current density plotted on the $x$-axis has been normalized by the concentration ratio, $X$, and is expressed as $J / X$. In a conventional single junction solar cell, the short circuit current under concentration has the relation, $J_{s c}(X)=X J_{s c}(1 \mathrm{sun})$, thus $J / X$ is constant and equal to $J_{s c}\left(1\right.$ sun). However, as can be seen in Figure 9, for IBSC, the relation of $J_{s c}(X)$ to $X$ is nonlinear and the ratio $J / X$ is increases with increase of $X$, until saturation at large $X$. In our recent work, we have demonstrated that the nonlinear relation between $J_{s c}(\mathrm{X})$ and $\mathrm{X}$ is one crucial fingerprint to evaluate the carrier dynamics via IB for IBSC serving under light concentration. ${ }^{24}$ Effect of non-radiative transition like electron-phonon interaction or Auger effects ${ }^{11,25}$ on the efficiency of such QD in QWR structures is yet to be examined both experimentally and theoretically.

In addition, it should be noted that the efficiency for a GaAs/AlGaAs QD is much lower than the ideal IBSC efficiency of $47 \%$ under 1 sun and $63 \%$ under full sun. This is due to the fact that the location of the IB in the current case is not optimal and the IB here is functioning as a recombination centre rather than an extra carrier generation centre. However, if we choose an appropriate material combination in order to create IB in the optimal position between CB and VB, the efficiency will be greatly improved and will approach the ideal value. One feasible

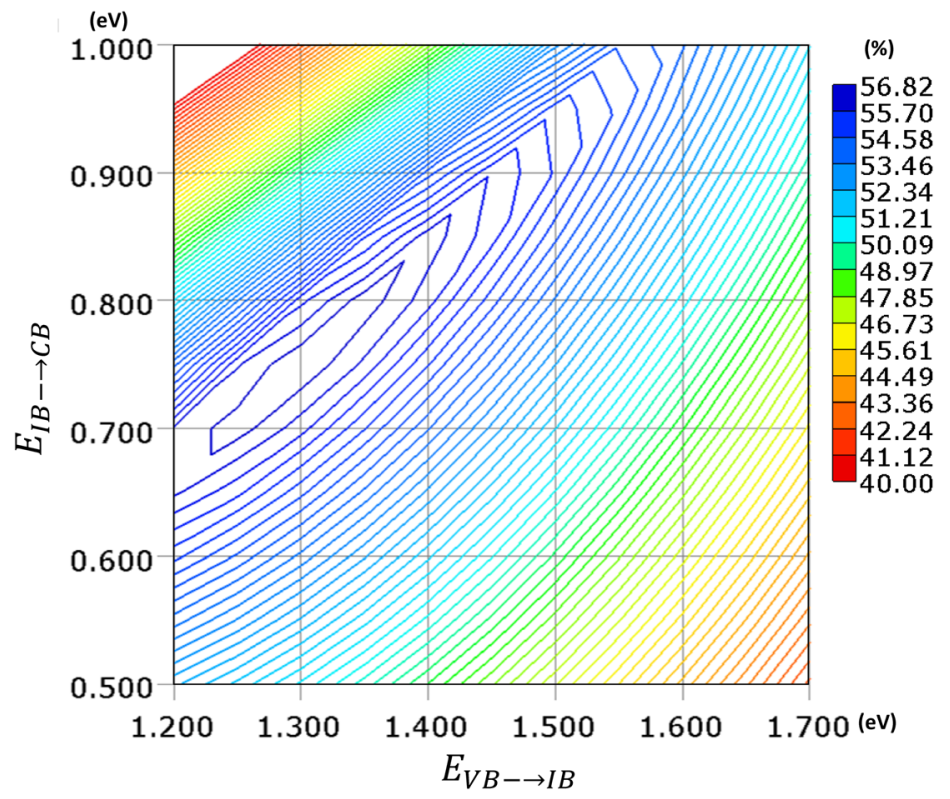

FIG. 8. Conversion efficiency contour map of IBSC calculated using the drift-diffusion model including the photofilling effect in IB. The contour plotted the relation by varying the energy separation between $\mathrm{VB} \rightarrow \mathrm{IB}$ and IB $\rightarrow \mathrm{CB}$. 


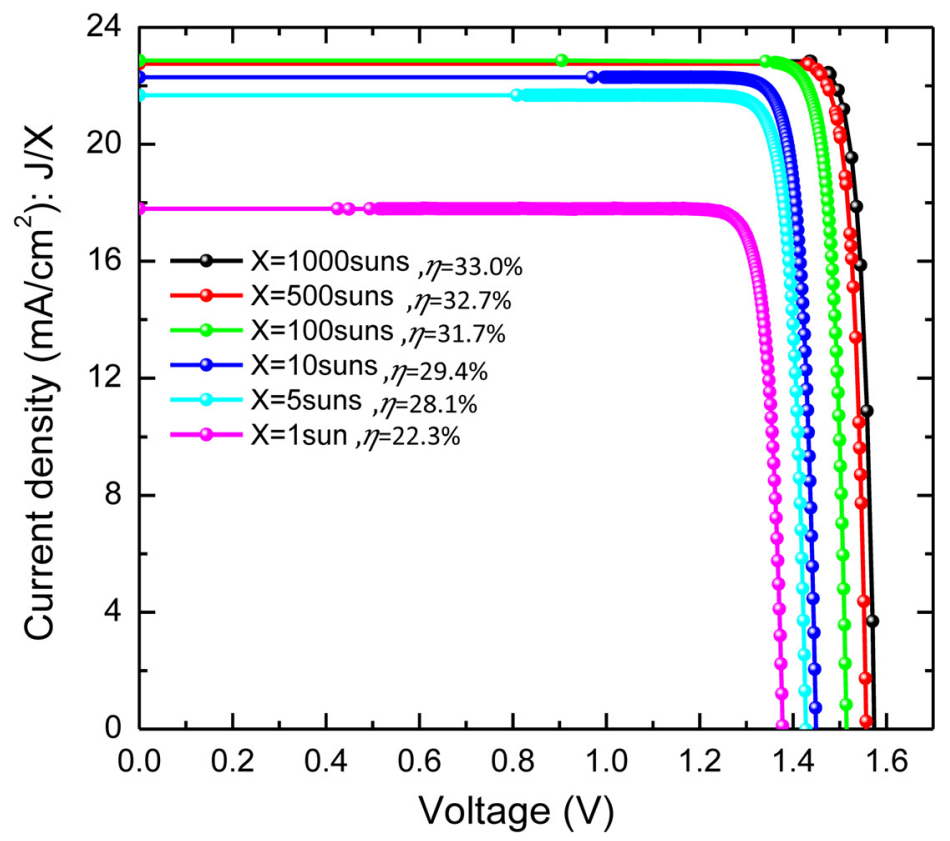

FIG. 9. I-V curves for GaAs QD based IBSC under different concentrate ratio. It must be noticed that the current density on the $y$-axis was normalised by the concentration ratio as: $J / X$. A clear nonlinear relation of $J \propto X$ is observed because the ratio of $J / X$ is varying with different $X$.

solution is to replace the GaAs QD with a lower band gap material such as InGaAs to lower down the position of the IB. Although there exists some issues such as lattice mismatch, using the lithographic and etching methods developed in our group will greatly reduce the difficulties as one need only to control the epitaxial growth of InGaAs QW/AlGaAs.

\section{CONCLUSIONS}

In conclusion, we have simulated an IBSC made of a GaAs QD array embedded in $\mathrm{AlGaAs}$ barrier material by combining a multiband $\mathbf{k} \cdot \mathbf{p}$ and drift-diffusion model adapted for IBSC. Detailed calculations of the optical absorption and spontaneous emission of both GaAs QW and GaAs QD were performed and the results were in good agreement with experimental photoluminescence results. Using a drift diffusion model adapted for IBSC, we obtained conversion efficiency of $22.3 \%$ for a GaAs QD based IBSC under 1 sun and 33\% under 1000 suns. The efficiency will be effectively improved by replacing the GaAs QD with a lower band gap material so as to lower the position of IB.

\section{ACKNOWLEDGMENTS}

The authors are grateful to the New Energy and Industrial Technology Development Organisation (NEDO), Japan, for financial support under grant: Research and Development on Innovative Solar Cells: Post-Silicon solar cells for ultra-high efficiencies. S.T. also wishes to thank the Royal Society, London, grant High Performance Computing in Modelling of Innovative PhotoVoltaic Devices, to the STFC e-Science, UK, for providing the computational resources and to M. Lundie for useful discussions.

${ }^{1}$ A. Luque and A. Martí, Phys. Rev. Lett. 78, 5014 (1997).

${ }^{2}$ A. Luque, A. Martí, and C. Stanley, Nature Photon. 6, 146 (2012).

${ }^{3}$ R. Oshima, A. Takata, and Y. Okada, Appl. Phys. Lett. 93, 083111 (2008).

${ }^{4}$ A. Luque, A. Martí, C. Stanley, N. López, L. Cuadra, D. Zhou, J. L. Pearson, and A. McKee, J. Appl. Phys. 96, 903 (2004).

${ }^{5}$ S. M. Hubbard, C. D. Cress, C. G. Bailey, R. P. Raffaelle, S. G. Bailey, and D. M. Wilt, Appl. Phys. Lett. 92, 123512 (2008). 
${ }^{6}$ S. Blokhin, A. Sakharov, A. Nadtochy, A. Pauysov, M. Maximov, N. Ledentsov, A. Kovsh, S. Mikhrin, V. Lantratov, S. Mintairov, N. Kaluzhniy, and M. Shvarts, Semiconductors 43, 514 (2009).

${ }^{7}$ S. Tomić, T. Sogabe, and Y. Okada, "In-plane coupling effect on absorption coefficients of InAs/GaAs quantum dots arrays for intermediate band solar cell," Progress in Photovoltaics (submitted).

${ }^{8}$ T. Kaizu, Y. Tamura, M. Igarashi, W. G. Hu, R. Tsukamoto, I. Yamashita, S. Samukawa, and Y. Okada, Appl. Phys. Lett. 101, 113108 (2012).

${ }^{9}$ S. Tomić, T. S. Jones, and N. M. Harrison, Appl. Phys. Lett. 93, 263105 (2008).

${ }^{10}$ R. Strandberg and T. W. Reenaas, J. Appl. Phys. 105, 124512 (2009).

${ }^{11}$ S. Tomić, Phys. Rev. B 82, 195321 (2010).

${ }^{12}$ A. D. Andreev, J. R. Downes, D. A. Faux, and E. P. O’Reilly, J. Appl. Phys. 86, 297 (1999).

${ }^{13}$ S. Tomić, A. G. Sunderland, and I. J. Bush, J. Mater. Chem. 16, 1963 (2006).

${ }^{14}$ S. Tomić, P. Howe, N. M. Harrison, and T. S. Jones, J. Appl. Phys. 99, 093522 (2006).

${ }^{15}$ E. Zielinski, F. Keppler, S. Hausser, M. H. Pilkuhn, R. Sauer, and W. T. Tsang, IEEE J. Quantum Electron. 25, 1407 (1989).

${ }^{16}$ S. L. Chuang, Physics of Photonic Devices (John Wiley and Sons, 2009).

${ }^{17}$ J. Kim and S. L. Chuang, IEEE J. Quantum Electron. 42, 942 (2006).

${ }^{18}$ S. Tomić, E. P. O'Reilly, R. Fehse, S. J. Sweeney, A. R. Adams, A. D. Andreev, S. A. Choulis, T. J. S. Hosea, and H. Riechert, IEEE J. Sel. Top. Quantum Electron. 9, 1228 (2003).

${ }^{19}$ A. Danicić, J. Radovanović, V. Milanović, D. Indjin, and Z. Ikonić, J. Phys. D: Appl. Phys. 43, 045101 (2010).

${ }^{20}$ D. Timotijević, J. Radovanović, and V. Milanović, Semicond. Sci. Technol. 27, 045006 (2012).

${ }^{21}$ L. Cuadra, A. Martí, and A. Luque, IEEE Trans. Electron Devices 51, 1002 (2004).

${ }^{22}$ M. J. Keevers and M. A. Green, J. Appl. Phys. 75, 4022 (1994).

${ }^{23}$ A. Luque, A. Martí, N. López, E. Antolín, E. Cánovas, C. Stanley, C. Farmer, and P. Díaz, J. Appl. Phys. 99, 094503 (2006).

${ }^{24}$ T. Sogabe, Y. Shoji, M. Ohba, K. Yoshida, H.-F. Hong, C.-H. Wu, C.-T. Kuo, S. Tomić, and Y. Okada, unpublished results (2013).

${ }^{25}$ S. Tomić, A. Marti, E. Antolin, and A. Luque, Appl. Phys. Lett. 99, 053504 (2011). 